\title{
Detection of a New Hainan Gibbon (Nomascus hainanus) Group Using Acoustic Call Playback
}

Jessica V. Bryant, 1 ,

Email jessica.bryant@ioz.ac.uk

Aurélien Brulé, 2

Michelle H. G. Wong,

Xiaojiang Hong, 3

Zhaoli Zhou, ${ }^{3}$

Wentao Han, ${ }^{3}$

Timothy E. Jeffree,

Samuel T. Turvey, 1

1 Institute of Zoology, Zoological Society of London, London, NW1 4RY UK

2 Project Kalaweit, Palangka Raya, Central Kalimantan, Kalimantan, Indonesia

3 Bawangling National Nature Reserve Management Office, Changjiang Lizu Autonomous County, Hainan, China

\section{Abstract}

Targeted management actions informed by robust data are needed to conserve species of extreme rarity, and assessing the effectiveness of different field methods for detection and monitoring of such species is a conservation priority. Gibbons are typically detected by their daily song through passive listening surveys, but lone gibbon individuals and low-density populations are less likely to sing, making detection difficult or impossible using standard survey techniques. Call playback represents an alternative potential method for detecting gibbon presence, but there has been no empirical evaluation of the usefulness of this method in the field. We investigated the efficacy of call 
playback as a survey method for detecting previously unconfirmed or unknown individuals of the Critically Endangered Hainan gibbon (Nomascus hainanus), the world's rarest primate, in patches of good-quality forest outside the current home ranges of the three known Hainan gibbon social groups in Bawangling National Nature Reserve, Hainan, China. Call playback led to detection of a male-only call likely to have been made by a solitary male, and a previously unknown social group comprising an adult male, adult female, and an infant, increasing the number of known breeding females in the global Hainan gibbon population from five to six. Call playback therefore represents an effective tool for improved monitoring of Hainan gibbons, as well as other gibbon populations; however, it is a moderately disruptive survey technique, and should be employed sparingly, in key locations, and for short periods of time only when attempting to detect gibbon presence.

\section{Keywords}

Critically Endangered

Gibbon

Nomascus hainanus

Population monitoring

Social group

Survey methods

\section{Handling Editor: Joanna M. Setchell}

\section{Introduction}

When highly threatened species are reduced to only a handful of surviving individuals, urgent, targeted, and intensive management actions are typically needed to conserve them, and delays can mean the difference between extinction and recovery (Grantham et al. 2009; Rabinowitz 1995; Turvey 2008). For conservation management to be most effective, decision making must be informed and guided by empirical data on a species' ecology, population dynamics, and threats. This is especially true for "species of extreme rarity" (Groombridge et al. 2004, p. 366), which comprise single remnant populations of very few individuals and no captive individuals/populations, and for which there is therefore no margin for error in management actions. However, the very rarity of such species can make them challenging to study, with robust data often difficult to obtain. Successful evidence-based conservation of species of extreme rarity may therefore necessitate using alternative investigative approaches to gain an accurate picture of their status and key population parameters. Identifying novel field methods and assessing their usefulness for effective 
detection and monitoring of such species is therefore an important conservation priority.

Gibbons are typically detected by their daily song during population surveys that use variants of the fixed point count survey method, whereby researchers listen opportunistically for calls at multiple elevated listening posts across the landscape (Brockelman and Ali 1987; Brockelman and Srikosamatara 1993). However, when population densities become low, the need to advertise territory and maintain group cohesiveness against rivals may be reduced, making groups potentially less likely to expend the energetic cost of singing, such that groups call less frequently at lower population densities (Brockelman et al. 1974; Chivers 1974; Nijman 2004). Solitary individuals are also very unlikely to call, and at most do so very infrequently, as this behavior can risk aggressive attacks by neighboring groups acting to protect their territories, which can lead to gibbon deaths (Susan Cheyne pers. comm. March 2014). This lack of regular calling makes detection of lone gibbon individuals and isolated populations extremely difficult or impossible using standard survey techniques for gibbons at low population densities.

One of the main functions of a gibbon group's daily songs is to communicate with nearby groups (Cowlishaw 1992), and gibbon groups will respond to nearby group calls, beginning their singing bouts in response (J. V. Bryant pers. $o b s$.). An alternative potential method for detecting gibbons in forest landscapes may therefore be the use of "call playback," the process of broadcasting a recorded call of a target species with the aim of eliciting a response from previously undetected conspecific individuals in the local area, either through direct response/reply call or investigation of the call source (James and Robertson 1985). Call playback has been used in wildlife surveys since the late 1950s, when recorded calls were employed to count game birds (Bohl 1956), and the technique is now widely used to improve the number of individuals detected per unit effort and obtain more accurate population estimates for many birds (Allen et al. 2004; Conway and Gibbs 2005; Johnson et al. 1981) and amphibians (Sung et al. 2005; Wassens 2008). Use of call playback in mammal surveys is less common ( $c f$. Downey et al. 2006), and although it has previously been used to study gibbons, this has predominantly involved investigation of mechanisms maintaining territoriality and monogamy and the functions of song in groups (Mitani 1984, 1985, 1987; Raemaekers and Raemaekers 1985 ), rather than as a technique for locating individuals. However, these studies demonstrated that mated wild gibbons respond to played calls by approaching the sound source, or eliciting an alarm call or male-female duet (Mitani 1984, 1985 , 1987, 1990), with female "great calls" being sung more frequently than usual (Raemaekers and Raemaekers 1985 ), suggesting that call playback may 
represent a useful method for detecting the presence of previously unknown gibbon groups in landscapes of interest. Solitary gibbon individuals may also be likely to investigate the source of gibbon calls, especially if these are broadcast in novel locations, and could be detected by teams of observers placed strategically around the call playback source.

The Critically Endangered Hainan gibbon (Nomascus hainanus) is the world's rarest ape, rarest primate, and arguably rarest mammal (IUCN 2015), and comprises a single surviving population numbering an estimated 25 individuals in Bawangling National Nature Reserve (BNNR), Hainan, China. At the time of this study, this remnant population was known to consist of only three social groups (groups A, B, and C) and an unknown but likely small number of solitary individuals; the BNNR Management Office (BNNRMO) estimates that the reserve currently contains four solitary individuals, based on encounters or calls heard by the gibbon monitoring team during routine survey activities (although see Deng et al. 2015 for an alternative estimate of the number of solitary individuals present in the reserve). The population was thought to contain only five females of breeding age, which have all produced offspring within the three established social groups (Bryant 2014; Fellowes et al. 2008). No captive Hainan gibbon individuals exist, meaning that the loss of the BNNR population would constitute the global extinction of the species.

To understand the status of the last surviving Hainan gibbon population and develop informed conservation actions to attempt to recover the species, it is crucial to obtain detailed information on the major population-level mechanisms likely to be affecting this small population, including survivorship, population recruitment, and rate of group formation. New gibbon social groups form when maturing subadults that have dispersed from their natal group form pair bonds with an animal of the opposite sex (MacKinnon and MacKinnon 1977). Hainan gibbon individuals reach sexual maturity at $c a$. 5-8 years of age, and exhibit ontogenetic and sexual dichromatism: subadult individuals of both sexes are black, and at sexual maturity males remain black while females become predominantly yellow (Liu et al. 1989). Although each of the three gibbon social groups at BNNR is the focus of regular monitoring efforts by BNNRMO, there is considerably less clarity about the history, long-term social dynamics, or fate of any specific individuals once they leave their natal groups, or about the number or distribution of solitary gibbons that exist in BNNR, as our existing knowledge of solitary Hainan gibbons is based entirely on rare opportunistic encounters that provide only an anecdotal understanding of the existence of these individuals. More robust information on the status of these solitary gibbons would greatly assist in understanding and predicting changes in Hainan gibbon social group size, composition, and dynamics, and potential dispersal events and 
the likelihood of new group formation. An improved understanding of gibbon dispersal and solitary gibbons in BNNR is particularly important because, despite formal protection measures and an apparently normal birth rate (Fellowes et al. 2008), the BNNR gibbon population has fluctuated between $c a .15$ and 25 individuals and shown no consistent growth for several decades (Bryant 2014; Turvey et al. 2015), suggesting that population recovery may be constrained by factors associated with gibbon dispersal and social group formation. The rate of group formation at BNNR is presently very low, possibly driven by a lack of availability and suitability of mates due to high levels of familiarity and relatedness in the remaining population (Bryant et al. 2016), a lack of space for new territory formation due to limited available habitat, low population density leading to post-dispersal difficulties in locating potential mates, or a combination of these factors (Turvey et al. 2015).

Development of additional detection methods is therefore required to strengthen the capacity for monitoring gibbons more effectively in BNNR, as well as in other situations where gibbon populations have become extremely low, including to assess the possible existence of remnant Hainan gibbon populations or individuals that may conceivably still survive elsewhere on Hainan (Turvey et al. 2015 ). Furthermore, identifying novel monitoring tools and generating a more robust evidence base on the fate of dispersing individuals have both been identified as priority activities for Hainan gibbon conservation research (Turvey et al. 2015). Although we might be able to predict possible gibbon responses to call playback, there has been no empirical evaluation of the usefulness of this method for surveying gibbon populations in the field. In this study, we therefore aimed to investigate the usefulness of call playback for detecting previously unconfirmed Hainan gibbons in BNNR by carrying out controlled playback trials at two locations outside the current recorded range of the known Hainan gibbon groups in BNNR. We hypothesized that if any previously unconfirmed Hainan gibbons were nearby to our call playback locations (within audible range) when the recorded call was broadcast, they would investigate the call payback source by moving closer to it and would thereby become detectable by our team of observers. Our ultimate objective was to evaluate the efficacy of this survey technique as a possible tool for improved monitoring of Hainan gibbons, a tool that may also be applicable to other populations of gibbons that have been reduced to extremely low densities.

\section{Methods}

We conducted our study over June 9-19, 2015, in BNNR $\left(18^{\circ} 57^{\prime}-19^{\circ} 11^{\prime} \mathrm{N}\right.$, $109^{\circ} 03^{\prime}-109^{\circ} 17^{\prime} \mathrm{E}$; Fig. 1). The reserve has a total area of almost $300 \mathrm{~km}^{2}$, but the surviving Hainan gibbon population is apparently restricted to a $c a .15 \mathrm{~km}^{2}$ 
area of relatively high-elevation fragmented forest $800-1200 \mathrm{~m}$ above sea level in the Futouling-Honghegu region of the reserve (Bryant 2014; Chan et al. 2005; Deng et al. 2015; Fellowes et al. 2008; Zhang et al. 2010). The Hainan gibbon is the only gibbon species present in BNNR or elsewhere on Hainan, and the only other primate species found in BNNR, the rhesus macaque (Macaca mulatta), does not sing (Mittermeier et al. 2013), so there is no risk of misidentification based on song. Furthermore, the majority of observers in the survey team have several years of experience monitoring the BNNR Hainan gibbon population and can accurately discriminate the different existing social groups based on their daily songs, so we are confident about their ability to identify the songs of any potential new individuals.

\section{Fig. 1}

Map of Bawangling National Nature Reserve, Hainan, China, showing current distribution of known Hainan gibbon social groups (home range shapefiles from Bryant 2014), and main geographic locations mentioned in the text that were used during fieldwork June 9-19, 2015.

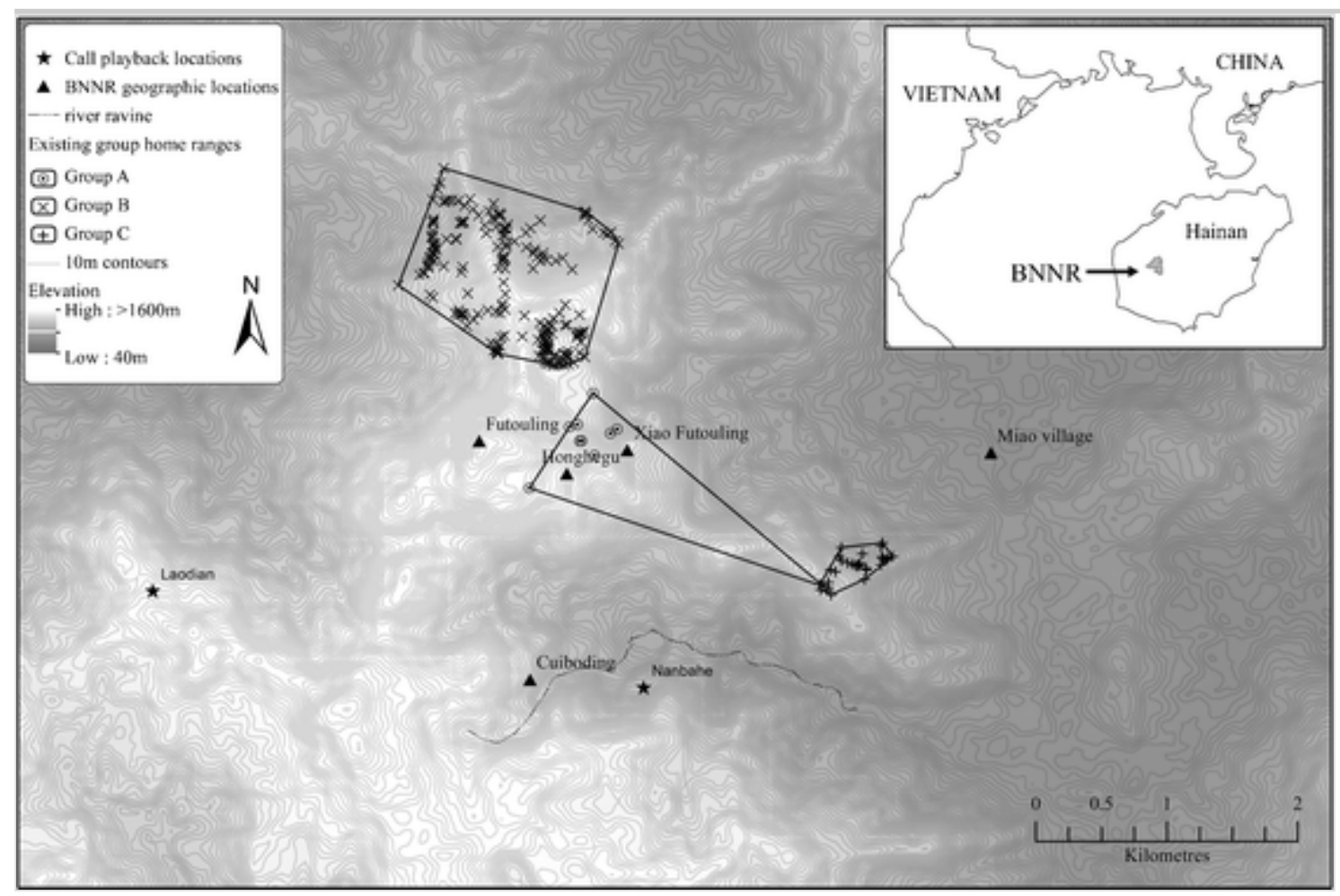

We selected two locations in BNNR, Nanbahe and Laodian, that are located $c a$. $3.5 \mathrm{~km}$ apart (Fig. 1), to investigate the efficacy of call playback as a survey method for detecting previously unconfirmed Hainan gibbon individuals. These locations were identified based on opportunistic encounters with lone gibbons outside the known recent home ranges of the three recorded social groups (Bryant 2014), reported periodically to the lead and senior authors by BNNR wardens and local community members during the course of longer-term 
conservation research at BNNR (Nash et al. 2016). Preliminary investigation of forest quality indicated that habitat at both sites was suitable to support gibbons and was comparable to forest occupied by gibbons in the Futouling-Honghegu region of the reserve.

\section{AQ1}

Nanbahe contains a patch of good-quality forest suitable for gibbons to the south of the current known range of the Hainan gibbon population in BNNR, but is separated by a large river ravine that is thought to act as a barrier to gibbon dispersal and range expansion from the Futouling-Honghegu area into forest further south in the reserve (BNNRMO pers. comm. August 2014). However, local informants reported that on at least one occasion an individual black, i.e., male or subadult, gibbon had been sighted recently in forest to the south of the ravine at Nanbahe. We therefore carried out call playback at this site to investigate whether any gibbon individuals may have crossed the ravine and dispersed into southerly forests in BNNR.

Laodian is located to the west of the current known range of the existing Hainan gibbon social groups in BNNR. Forest in this area is contiguous with that occupied by the existing population in the Futouling-Honghegu area; however, none of the social groups have been reported to range here in recent years, possibly owing to disturbance by humans associated with a 2-km-long wooden visitor boardwalk leading to "Five Finger Tree" (a large individual of Dacrydium pectinatum of local cultural significance) and constructed in the forest at this site in 2008 by the Hainan Forestry Bureau. However, there have been several independent but unverified reports from local informants of either a black gibbon or a yellow (adult female) gibbon being observed in this area on separate occasions. Owing to uncertainty over exact sighting dates, it is not possible to determine whether these putative reports represent a single maturing solitary female gibbon undergoing ontogenic change from black to yellow pelage, or multiple gibbon individuals potentially of both sexes; because of the proximity of Laodian to the territories of the three existing social groups, it is also unclear whether these supposed sightings represent known individuals from an existing group exploring areas close to their established territory, rather than one or more already dispersed solitary individuals.

After locating the general area in which gibbons had reportedly been encountered based on information from local informants, we selected an elevated location at each locationsite to conduct call playback. We preferentially chose slopes that were exposed to early morning sunlight rather than slopes that would remain in shadow for several hours after sunrise, which would be likely to be too cold for gibbons in the early morning. At each location, we fixed a FOXPRO 
Hellfire (FOXPRO Inc., Lewistown, PA, USA) portable speaker to a tree using bungee straps at a height of $\geq 2 \mathrm{~m}$ from the ground. One survey team member remained close to the speaker to control the playback, and four to six additional team members dispersed from this central location, with these team members moving $\geq 50 \mathrm{~m}$ from the speaker source in a different direction to cover all main axes. The peak Hainan gibbon singing period is 06:00-07:00 h, with singing continuing at decreasing regularity later into the morning and afternoon (Chan et al. 2005 ). We therefore commenced call playback at 05:30 h, half an hour before the expected start time of normal calling behavior, to avoid overlap with any calls from established gibbon groups in the vicinity for this period at least, and so maximize the chance of any nearby gibbons hearing our playback. The playback scheme consisted of playing a male-female duet call, previously recorded from group B by J. V. Bryant in 2011 using a Zoom H2 Handy Recorder (Zoom Corporation, Tokyo, Japan; using a two-channel setting with a sampling rate of $48 \mathrm{kHz}$, a 16-bit resolution, and high gain level), on a cycle of 15 min of playback interspersed with 5 min of silence. The duet recording was broadcast using volume step 20 on the FOXPRO Hellfire (maximum possible volume for the speaker, $c a .120$ decibels). Call playback continued until 11:00 h (by which time normal Hainan gibbon calling behavior becomes infrequent), or until gibbons were detected, whichever occurred first.

During call playback the survey team constantly scanned the canopy for signs of gibbons, and during the silent periods we conducted both visual scans and listened for any vocalizations (group calls, solo calls, or alert/alarm calls). If any team member detected any signs of a gibbon, he or she attempted to locate and maintain visual contact long enough to confirm crucial details (number of individuals, color /sex, approximate age), then returned to the call playback source to inform the speaker operator to cease call playback. We allowed a period of 5 days to carry out call playback at each site, as previous field studies of other gibbon species suggest that call playback for at least a week is likely to attract new gibbon groups to investigate (Turvey et al. 2015). We conducted call playback first on a slope above the ravine at Nanbahe, in a forest patch on the south side of the ravine opposite the area occupied by existing gibbon social groups, and then at the very end of the Laodian boardwalk. During call playback at Laodian, we also simultaneously monitored the three existing social groups to ensure that any gibbons detected were not known individuals that had strayed temporarily from one of these groups. The day before commencement of call playback work at Laodian, an additional three separate survey teams located each existing social group and continuously tracked each group during this phase of call playback, with all four teams in constant communication. 


\section{Ethical Note}

Research conducted in this study complied with protocols approved by the Hainan Forestry Bureau and the Hainan Provincial Government, and adhered to the legal requirements of the People's Republic of China. Project design was approved by the Zoological Society of London's Ethics Committee.

\section{Results}

Conditions remained dry throughout the call playback surveys at both sites, with a daily mean high temperature of $33.6{ }^{\circ} \mathrm{C}$ and mean low temperature of $28.8{ }^{\circ} \mathrm{C}$. On the second day of call playback at Nanbahe (June 12, 2015), we detected a male sequence at 08:26 h from the Cuiboding region on the north side of the ravine. This sequence was brief (lasting $c a .30 \mathrm{~s}$ ) and was heard only once and by only two members of the team, who estimated the individual to be $<1 \mathrm{~km}$ away from their location. However, it was clear that this was not the call of an existing social group, because groups A and B were known to be too far away at the time to be heard from this location, and group $\mathrm{C}$ was heard to duet shortly afterwards ( $c a .20 \mathrm{~min}$ later, for a duration of $c a .15 \mathrm{~min}$ ) in a different location on the north side of the ravine, close to the Xiao Futouling and Miao village region that they typically occupy; the call was therefore most likely to have been made by a previously undetected solitary male individual. The exact distance between group $\mathrm{C}$ and the solitary male was unclear, but as we could hear this group, it is also likely that the group could hear the solitary male and the duet used for call playback. Solitary gibbons that sing can be at risk of aggressive attacks by neighboring groups acting to protect their territory (Susan Cheyne pers. comm. March 2014); given the proximity of this individual to group C, and the extreme rarity of the species, we decided to exercise maximum caution and cease call playback at this point to prevent any risk of a potential aggressive attack by group C. We attempted to locate the solitary individual by carrying out passive listening post monitoring without call playback in the Cuiboding area for an additional day, but we detected no visual or auditory signs of this individual.

On the second day of call playback at Laodian (June 17, 2015), we detected another male-only call, which consisted of two initial call bouts $c a .25$ min apart (07:41-07:59 h, and 08:26-08:55 h) that were heard by all team members. On detection of these calls, we made the decision that one team member should to try to locate the group, to keep disturbance to a minimum and therefore maximize the chance of observing the male individual. The team also made contact with the other teams tracking groups $\mathrm{A}, \mathrm{B}$, and $\mathrm{C}$ and established that known individuals in all three groups were accounted for, confirming that the male at Laodian was a previously unconfirmed individual. The rest of the team members remained at their stations and continued to scan for gibbons, with call 
playback continued on a revised schedule of silence for 30 min followed by 15 min of broadcast of the male-female duet.

At around 09:00 h, the observer made brief visual contact (lasting $<1 \mathrm{~min}$, at a distance of $c a .25 \mathrm{~m}$ ) with a black gibbon, estimated to be a young adult male $c a$. $8-10$ years in age. The gibbon moved away shortly after this initial observation, and although it did not appear nervous and apparently did not notice the observer during this brief visual contact, it was not followed to avoid disturbing the individual. At 10:14 h, the male gibbon called for a third time, close to the location at which contact had been lost with it. Using this call, the observer was able to relocate the male and made visual contact again ( $c a .5$ min into the calling bout), and also observed a young adult female carrying a black infant estimated at $c a$. 4-6 months of age (Fig. 2). The male call ceased at 10:25 h, ca. 5 min after visual contact was made and 11 min after the calling bout commenced, at which point both adult gibbons began to make alert calls after detecting the observer's presence. After the observer took steps to minimize his presence and stature (covering the face, and assuming a stationary, horizontal, nonthreatening position on the forest floor), the male and female gibbons became quiet and ceased alert calling at $c a .10: 50 \mathrm{~h}$. The gibbons also made no attempt to move away from the observer, instead becoming curious and making efforts to observe him. The observer was then able to observe the gibbons for $c a$. $1 \mathrm{~h}$ at a distance of $c a .15 \mathrm{~m}$, during which he was able to confirm that the three individuals constituted an established social group, with the male and female remaining in close proximity ( $<20 \mathrm{~m}$ from each other), and the black infant remaining attached to the female for the duration of the visual contact. No further individuals appeared during the visual contact, indicating that the three observed individuals were the only members of this social group.

\section{Fig. 2}

Female and infant individuals from a fourth, previously unknown Hainan gibbon social group, group D, first detected at Laodian, Bawangling National Nature Reserve, on June 17, 2015. 

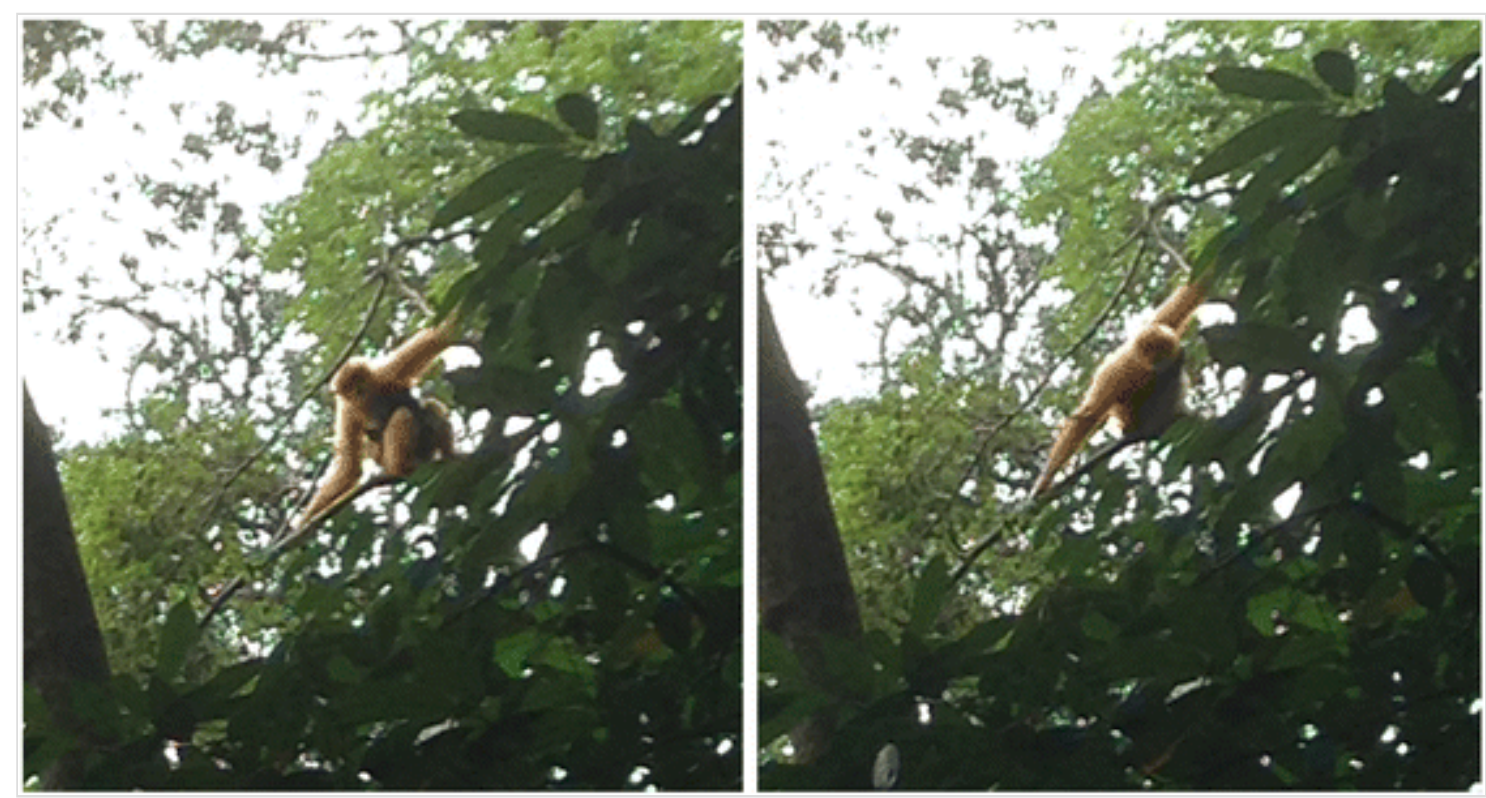

\section{Discussion}

By using call playback to promote a response and so enhance detectability of gibbons in the landscape, we were able to both detect a solitary individual and locate a previously unknown social group for the Hainan gibbon, the world's rarest primate. Our discovery of a new fourth Hainan gibbon social group (group D) in the Laodian area, comprising an adult male, adult female, and infant, increases the number of social groups known for the species in BNNR. The detection of both group $\mathrm{D}$ and the solitary individual at Cuiboding also increases the number of known Hainan gibbon individuals from $c a$. 25 individuals to at least 26, because of the new infant in group D, and possibly up to as many as 29 if all of the other individuals are also previously unknown; however, it is unfortunately very difficult to determine whether any, some, or all of these adult gibbons represent individuals that were previously accounted for as solitary individuals in estimates of Hainan gibbon population size by BNNRMO. Perhaps more significantly, the new group increases the number of known breeding females in the global Hainan gibbon population from five to six, representing a $20 \%$ increase in the reproductive potential of the last surviving population of the species. Although the new group did not perform a duet call, with the male instead calling alone possibly because of the group's youth or potential lack of a clear territory boundary, the presence of an infant suggests that it is socially stable and has sufficient resources to reproduce. This is an extremely positive sign, as it suggests that suitable habitat is available in BNNR to support additional gibbon social groups outside the current range of the three previously known groups; this result is particularly encouraging for the recovery and longterm survival of this Critically Endangered species. 
Our study represents a first attempt at assessing the efficacy of targeted call playback to improve detection of previously unconfirmed or poorly known gibbons. We set out to assessevaluate the usefulness of this method to improve detection of solitary gibbon individuals, as these are the hardest to detect using standard methods, and our detection of a solitary male Hainan gibbon at Nanbahe indicates that call playback can indeed be used to locate solitary gibbons and can even prompt a call response from such individuals. Our discovery of a new fourth Hainan gibbon social group was a very welcome but unexpected outcome of this study, and demonstrates that call playback can also be used to locate gibbon groups. The success achieved here indicates that call playback can be an informative survey method for enhancing gibbon population monitoring, by providing direct, systematic evidence of additional gibbons outside known social groups in a population, when it is employed using a strategy of simultaneous monitoring of existing social groups. Call playback therefore represents a promising supplementary tool that may be applicable for all gibbon species, including those existing at low population densities.

However, there has been only limited use of playback for gibbons to date (Mitani $1984,1985,1987)$, and the extent of the disturbance it may cause to groups and individuals is not yet fully known. As call playback uses a stimulus to promote a behavioral response by any gibbons near to the sound source, it may prove costly for both groups and solitary individuals by preventing them from behaving and ranging as normal, and may therefore potentially produce missed opportunity costs (Francis and Barber 2013). Conversely, it may be that if a call is broadcast for too long or too frequently, gibbons may identify the source or even become habituated to the stimulus (Mitani 1985), which may reduce the efficiency of the technique. More seriously, using call playback to detect solitary gibbons may attract gibbon social groups ranging nearby, which could lead to aggressive encounters with solitary individuals and therefore risks to solitary gibbon safety if they are both drawn to the source, as gibbon groups have been found to exhibit marked agonistic tendencies toward simulated conspecific intruders when playback is employed (Mitani 1990).

Because of these associated risks, call playback should therefore be regarded as a moderately disruptive survey technique, and other potential negative effects also need to be investigated before this method can be implemented for other gibbon populations. Additional trials are required in which the call type (duets, male and female solos, alarm calls), broadcast volume, and regularity and respective duration of both the broadcast and silent periods should be varied to determine the most effective but least disruptive procedure, whereby a balance can be stuck between the risks involved in using this technique and the benefits to be gained. Trials for other gibbon species under different site conditions, e.g., flat vs. 
mountainous landscapes, high vs. low gibbon population densities, are also required before a robust, detailed protocol can be developed that is more generally applicable to all gibbon species/populations, although it may be that only genus-specific or even species-specific protocols are possible and appropriate, as appears to be the case for owls in North America (Francis and Bradstreet 1997; Takats et al. 2001) and frogs in Australia (DEWHA 2010). Until these aspects can be resolved, call playback should be employed sparingly as a specialized tool, in key locations (based on recent or past evidence of gibbon occurrence, or presence of gibbon-appropriate habitat), and for only short periods of time (up to 1 week) when attempting to detect the presence of unknown, unconfirmed, or cryptic gibbon individuals. Furthermore, this technique should not be used as a tool for continuous, daily population monitoring, but instead should be employed only to supplement traditional fixedpost passive listening surveys when information becomes available on possible undetected individuals or groups at specific locations.

We propose that call playback can support improved long-term monitoring of the known Hainan gibbon population in BNNR, together with potential detection of other surviving gibbons both across the BNNR landscape and more widely across Hainan. If used in tandem with detailed information on the composition and individual identification for each existing social group, call playback also has the capacity to improve our understanding of the fate of specific dispersing individuals by increasing the detectability of newly formed social groups, via promotion of a call response (either duet or solo sequence). The ability of this method to enhance the detectability of solitary gibbons is, however, less conclusive, and further tests are required to ascertain if this is an efficient method for finding lone gibbons (in comparison to other detection methods, such as reports from local informants). A more systematic and robust monitoring program for the Hainan gibbon that includes call playback could, however, have the potential to generate more detailed information on long-term social dynamics of the population, which can hopefully be used to better understand and predict changes in social group size, composition, and dynamics, and potential dispersal events and the likelihood of new social group formation. Such information would also allow the impact of management actions to be better-determined, and could provide a more responsive early warning signal if a sudden population decline were to occur (Turvey et al. 2015).

\section{Recommendations and Required Actions}

In light of our discovery of group D at Laodian and detection of a solitary male gibbon at Nanbahe, and in line with the Hainan gibbon conservation priorities of generating a more robust evidence base on the fate of dispersing gibbon 
individuals and strengthening the capacity for monitoring the existing gibbon population, particularly solitary individuals (Turvey et al. 2015), we advocate the following four priority actions for the Hainan gibbon population at BNNR. These actions will provide further insights into the status and behavior of the newly detected gibbons as well as an improved understanding of existing gibbon social group compositions and dynamics at BNNR, which will ultimately enhance our ability to predict potential dispersal events and the likelihood of new group formation for the Hainan gibbon population. Such information is crucial to elucidating the major population-level mechanisms likely to be affecting this small population, including survivorship, population recruitment, and rate of group formation.

1. Systematically monitor group D. Beyond simply detecting group D, developing a better understanding of this social group is a key priority, and we recommend that the group's home range and interactions with other existing gibbon groups in BNNR are both investigated, together with potential changes in group composition over time. It is possible that the group's range is not centered on Laodian, as call playback may have drawn the gibbons away from the core of their territory to investigate the call source. BNNRMO has conducted monitoring at Laodian every month since the group's discovery and has regularly detected gibbons at the site; J. V. Bryant also heard a male gibbon singing at Laodian at 09:30 h during a short repeat visit on February 1, 2016. We recommend the continued use of simple passive listening-post monitoring (at least once/month) to track the group's location, movement, and behavioral dynamics with minimum disturbance.

2. Confirm the solitary male at Nanbahe. It is vital to determine if the male call detected during call playback at Nanbahe was definitely made by a solitary male; in light of our discovery of the nondueting group D at Laodian, this male may conceivably also represent a nondueting group. Additional surveys beyond call playback are therefore necessary at Cuiboding, for which we recommend the use of both simple passive listening post monitoring and more intensive but noninvasive monitoring methods such as camera trapping, which will maximize likelihood of detection but minimize disturbance to gibbons.

3. Improve monitoring of all established social groups. Our call playback trials demonstrated that it is possible to detect additional social groups using this method. However, once detected, all established groups must be carefully monitored to strengthen our understanding of the status and interactions of existing social groups, and therefore improve detection and 
understanding of solitary individuals dispersed across the BNNR landscape. It is therefore crucial that current gibbon monitoring methods at BNNR are enhanced. We strongly recommend that all existing groups are monitored more intensively, with a proposed monitoring strategy of continuous all-day tracking from first call to settling in sleeping trees, for a period of at least $1 \mathrm{month} /$ year. Conducting intensive monitoring at approximately the same time each year would enable BNNRMO to collect vital information on each group, including number, identity, approximate age and sex of individuals in each group, loss of any individuals from groups, approximate territory of each group, and interactions between groups. Recording and updating this information on an annual basis would enable development of an identification guide for all individuals in the remaining population and permit key population dynamics to be better understood for the overall gibbon population at BNNR, including changes in group composition, maturation of individuals, dispersal events, and identification of the origin of solitary individuals.

\section{Minimize disturbance at Laodian. If the Hainan gibbon population is to} expand beyond its current extent, then disturbance in all existing areas of the BNNR landscape with contiguous good-quality forest needs to be minimized as a priority (Chan et al. 2005; Fellowes et al. 2008; Turvey et al. 2015; Zhang et al. 2010). Forest in Laodian likely represents prime habitat for gibbons, is contiguous with forest in the Futouling-Honghegu region, which supports the other three known social groups, and could likely support more than one gibbon group. However, group D was previously unknown, having not been detected by any recent systematic surveys (Turvey et al. 2015), and is therefore likely to be a recently formed group and as such very vulnerable to disturbance. Detection of this group at Laodian means that this area should now be designated as part of the core area of the reserve, and requires a higher level of protection. We therefore recommend that access to Laodian should be strictly limited. Although the Laodian boardwalk has officially been closed to the public for 3 years, it continues to attract visitors; our survey team observed two separate tourist groups during the time spent at the site conducting our call playback work. We strongly urge BNNRMO and the Hainan Forestry Bureau (who are responsible for management of the boardwalk) to ensure that measures are taken to remove all nonessential disturbance to this new group, in particular tourist activity at the site, which may well deter gibbons from the area. To safeguard group D and increase suitability of the area for other potential gibbon groups, we therefore recommend that BNNRMO should close the Laodian boardwalk to the publicall visitors (except monitoring teams), or at 
least partially close the boardwalk or reduce its length and strictly limit access to the site.

\section{Conclusions}

This work constitutes an important case study that demonstrates the potential of call playback as an additional technique in the conservation toolkit for threatened primates. However, whether the call playback method is applicable more generally to other primate taxa remains to be seen. While gibbons' singing behavior makes them particularly appropriate for detection via call playback because of their call length and regularity, shorter vocalizations from other primate taxa, e.g., alarm calls, hoots, may also be appropriate for call playback protocols, as is the case for owl call playback surveys, which often use short (two-note) vocalizations (SOME 2014). We encourage further investigation of call playback across a wider range of primate taxa to permit assessment of the applicability and efficacy of this method for primates more broadly, particularly for highly threatened species.

\section{Acknowledgments}

This study was made possible through collaboration with BNNRMO, the Hainan Forestry Bureau (HFB), and the Hainan Provincial Forestry Department. We thank BNNRMO and HFB staff for assistance in the field. We are also grateful to the editor and two anonymous reviewers for their useful comments, which helped to improve the manuscript. Funding was provided by the Arcus Foundation, and a Royal Society University Research Fellowship (UF080320/130573) to S. T. Turvey.

\section{References}

Allen, T., Finkbeiner, S. L., \& Johnson, D. H. (2004). Comparison of detection rates of breeding marsh birds in passive and playback surveys at Lacreek National Wildlife Refuge, South Dakota. Waterbirds, 27, 277-281.

Bohl, W. H. (1956). Experiments in locating wild chukar partridges by use of recorded calls. Journal of Wildlife Management, 20, 83-85.

Brockelman, W. Y., \& Ali, R. (1987). Methods of surveying and sampling forest primate populations. In C. W. Marsh \& R. A. Mittermeier (Eds.), Primate conservation in the tropical forest (pp. 23-62). New York: Alan R. Liss. 
Brockelman, W. Y., Ross, B. A., \& Pantuwatana, S. (1974). Social interactions of adult gibbons (Hylobates lar) in an experimental colony. Gibbon and Siamang, 3, 137-156.

Brockelman, W. Y., \& Srikosamatara, S. (1993). Estimation of density of gibbon groups by use of loud songs. American Journal of Primatology, 29, 93-108.

Bryant, J. V. (2014). Developing a conservation evidence-base for the Critically Endangered Hainan gibbon (Nomascus hainanus). $\mathrm{PhD}$ thesis, University College London/Zoological Society of London.

Chan, B. P. L., Fellowes, J. R., Geissmann, T., \& Zhang, J. (2005). Hainan gibbon status survey and conservation action plan. Hong Kong: Kadoorie Farm \& Botanic Garden.

Chivers, D. J. (1974). The Siamang in Malaya: A field study of a primate in tropical rainforest. Contributions to Primatology, Vol. 4. Basel: Karger.

Conway, C. J., \& Gibbs, J. P. (2005). Effectiveness of call-broadcast surveys for monitoring marsh birds. Auk, 122, 26-35.

Cowlishaw, G. (1992). Song function in gibbons. Behaviour, 121, 131-153.

Deng, H., Zhang, M., \& Zhou, J. (2015). Recovery of the Critically Endangered Hainan gibbon Nomascus hainanus. Oryx. doi: $10.1017 / \mathrm{S} 0030605315000678$.

DEWHA. (2010). Survey guidelines for Australia's threatened frogs: Guidelines for detecting frogs listed as threatened under the EPBC Act. Barton: Australian Capital Territory: Department of the Environment, Water, Heritage and the Arts.

Downey, B. A., Jones, P. F., Quinlan, R. W., \& Scrimgeour, G. J. (2006). Use of playback alarm calls to detect and quantify habitat use by Richardson's ground squirrels. Wildlife Society Bulletin, 34, 480-484.

Fellowes, J. R., Chan, B. P. L., Zhou, J., Chen, S., \& Yang, S. (2008). Current status of the Hainan gibbon (Nomascus hainanus): Progress of population monitoring and other priority actions. Asian Primates Journal, 1, 2-9. 
Francis, C. D., \& Barber, J. R. (2013). A framework for understanding noise impacts on wildlife: an urgent conservation priority. Frontiers in Ecology and the Environment, 11, 305-313.

Francis, C. M., \& Bradstreet, M. S. W. (1997). Monitoring boreal forest owls in Ontario using tape playback surveys with volunteers. In J. R. Duncan, D. H. Johnson, T. H. Nicholls (Eds.), Biology and conservation of owls of the Northern Hemisphere: 2nd International Symposium (pp. 175-184). General Technical Report. St. Paul, MN: U.S. Department of Agriculture.

Grantham, H. S., Wilson, K. A., Moilanen, A., Rebelo, T., \& Possingham, H. P. (2009). Delaying conservation actions for improved knowledge: how long should we wait? Ecology Letters, 12, 293-301.

Groombridge, J. J., Massey, J. G., Bruch, J. C., Malcolm, T., Brosius, C. N., et al. (2004). An attempt to recover the po'ouli by translocation and an appraisal of recovery strategy for bird species of extreme rarity. Biological Conservation, 118, 365-375.

IUCN. (2015). IUCN Red List of Threatened Species. Version 2015-4. www.iucnredlist.org (Accessed October 6, 2015).

James, P. C., \& Robertson, H. A. (1985). The use of playback recordings to detect and census burrowing seabirds. Seabird, 8, 18-20.

Johnson, R. R., Brown, B. T., Haight, T., \& Simpson, J. M. (1981). Playback recordings as a special avian censusing technique. Studies in Avian Biology, 6, $68-75$.

Liu, Z., Zhang, Y., Jiang, H., \& Southwick, C. (1989). Population structure of Hylobates concolor in Bawanglin Nature Reserve, Hainan, China. American Journal of Primatology, 19, 247-254.

MacKinnon, J., \& MacKinnon, K. (1977). The formation of a new gibbon group. Primates, 18, 701-708.

Mitani, J. C. (1984). The behavioral regulation of monogamy in gibbons (Hylobates meulleri). Behavioural Ecology and Sociobiology, 15, 225-229.

Mitani, J. C. (1985). Gibbon song duets and intergroup spacing. Behaviour, 92, 59-96. 
Mitani, J. C. (1987). Territoriality and monogamy among agile gibbons (Hylobates agilis). Behavioural Ecology and Sociobiology, 20, 265-269.

Mitani, J. C. (1990). Experimental field studies of Asian ape social systems. International Journal of Primatology, 11, 103-126.

Mittermeier, R. A., Rylands, A. B., Wilson, D. E. (2013). Handbook of the mammals of the world, Vol. 3: Primates. Barcelona: Lynx Edicions.

Nash, H. C., Wong, M. H. G., \& Turvey, S. T. (2016). Using local ecological knowledge to determine status and threats of the Critically Endangered Chinese pangolin (Manis pentadactyla) in Hainan, China. Biological Conservation, 196, 189-195.

Nijman, V. (2004). Conservation of the Javan gibbon Hylobates moloch: Population estimates, local extinctions and conservation priorities. The Raffles Bulletin of Zoology, 51, 271-280.

Rabinowitz, A. (1995). Helping a species go extinct: the Sumatran rhino in Borneo. Conservation Biology, 9, 482-488.

Raemaekers, J. J., \& Raemaekers, P. W. (1985). Field playback of loud calls to gibbons (Hylobates lar): territorial, sex-specific and species-specific responses. Animal Behaviour, 33, 481-493.

SOME. (2014). Burrowing owl survey protocol. Fish and Wildlife Branch Technical Report No. 2014-05.. Regina: Saskatchewan Ministry of Environment.

Sung, H. C., Kim, S. K., Park, S. R., \& Park, D. S. (2005). Effectiveness of mating call playbacks in anuran call monitoring: a case study of three-striped pond frogs (Rana nigromaculata). Integrative Biosciences, 9, 199-203.

Takats, D. L., Francis, C. M., Holroyd, G. L., Duncan, J. R., Mazur, K. M., et al. (2001). Guidelines for nocturnal owl monitoring in North America. Edmonton: Beaverhill Bird Observatory and Bird Studies Canada.

Turvey, S. (2008). Witness to extinction: How we failed to save the Yangtze River dolphin. Oxford: Oxford University Press.

Turvey, S. T., Traylor-Holzer, K., Wong, M. H. G., Bryant, J. V., Zeng, X., et al. (2015). International conservation planning workshop for the Hainan 
gibbon: Final report. London: Zoological Society of London /IUCN SSC Conservation Breeding Specialist Group.

Wassens, S. (2008). Review of the past distribution and decline of the southern bell frog Litoria raniformis in New South Wales. Australian Zoologist, 34, 446-452.

Zhang, M., Fellowes, J. R., Jiang, X., Wang, W., Chan, B. P. L., et al. (2010). Degradation of tropical forest in Hainan, China, 1991-2008: conservation implications for Hainan gibbon (Nomascus hainanus). Biological Conservation, 143, 1397-1404.

J. V. Bryant, D. Gottelli, X. Zeng, X. Hong, B. P. L. Chan, J. R. Fellowes, Y. Zhang, J. Luo, C. Durrant, T. Geissmann, H. J. Chatterjee, S. T. Turvey, (2016) Assessing current genetic status of the Hainan gibbon using historical and demographic baselines: implications for conservation management of species of extreme rarity. Molecular Ecology 25 (15):3540-3556 